\title{
O ESTUDO DA PROVA CALÓRICA EM PACIENTES IDOSO
}

\author{
The study of caloric testing in senior patients
}

\author{
Débora Cristina de Oliveira Bezerra ${ }^{(1)}$, Silvana Frota ${ }^{(2)}$
}

\begin{abstract}
RESUMO
Objetivo: realizar o estudo da prova calórica em pacientes idosos com queixa de tontura, acompanhada ou não de zumbido com finalidade de traçar um perfil dos idosos quanto os sintomas vestibulares. Métodos: fizeram parte da amostra 143 pacientes, com faixa etária variando de 60 a 90 anos de idade; todos realizaram o exame otoneurológico. Foram excluídos pacientes idosos com queixa de desequilíbrio e aqueles que apresentaram a queixa de zumbido desacompanhado da tontura. Resultados: o estudo demonstrou que a normorreflexia, em valores absolutos, encontra-se entre $82,3 \%$ a $91,2 \%$ e os valores relativos são de $93 \%$. Referente aos dados colhidos na anamnese, o que chamou a atenção, foi que do total de pacientes analisados, encontrou-se a tontura acompanhada de zumbido em $69 \%$ dos casos e o elevado uso de medicação em pacientes nesta faixa etária. Conclusão: na população estudada houve um predomínio da tontura acompanhada de zumbido. Em relação à prova calórica, o trabalho demonstrou a alta prevalência de pacientes com valores absolutos e relativos normais.
\end{abstract}

DESCRITORES: Tontura; Vertigem; Idoso; Testes de Função Vestibular; Zumbido

\section{INTRODUÇÃO}

A tontura é um dos sintomas mais comuns no mundo em ambos os sexos, presente em mais de $10 \%$ da população mundial. Pode acometer crianças e adolescentes, porém é mais freqüente em adultos e idosos. Mais de $40 \%$ dos adultos relatam sua ocorrência em alguma época de suas vidas, acometendo principalmente indivíduos idosos.

A definição de tontura é a sensação de perturbação do equilíbrio corporal enquanto que a vertigem é uma sensação de desorientação espacial do tipo rotatório ${ }^{1}$. A vertigem sempre indica um desequilíbrio dentro do sistema vestibular, apesar do sintoma propriamente dito não especificar em que parte do sistema este desequilíbrio se origina ${ }^{2}$.

A manutenção do equilíbrio corporal estável no meio ambiente é determinado pela integração funcional das informações provenientes das estruturas sensoriais do sistema vestibular, visual e proprio-

(1) Fonoaudióloga; Clínica Sinus Center; Especialista em Audiologia pelo CEFAC - Saúde e Educação do Rio de Janeiro.

(2) Fonoaudióloga; Professora do CEFAC - Saúde e Educação e da Universidade Federal do Rio de Janeiro; Doutora em Distúrbio da Comunicação Humana pela Universidade Federal de São Paulo. ceptivo nos núcleos vestibulares do tronco encefálico, sob a coordenação do cerebelo ${ }^{1}$.

As tonturas podem ser relatadas como único sintoma, mas muitas vezes também são acompanhadas por outros sintomas e sinais, como diversos tipos de alterações auditivas e distúrbios neurovegetativos ${ }^{3}$.

Outra queixa que acomete indivíduos, principalmente idosos, é o zumbido. Existem várias classificações para o zumbido, porém o mais relatado na literatura é o que divide este em: subjetivo e objetivo. O zumbido subjetivo é aquele no qual só o paciente consegue percebê-lo e, no objetivo ele é percebido tanto pelo paciente quanto pelo examinador ${ }^{4}$.

Outra classificação utilizada é a que divide o zumbido quanto a sua fonte de origem em periótico (paraauditivos) e neurossensorial (auditivos). $O$ zumbido periótico se origina das estruturas musculares e/ou vasculares próximas à orelha interna. $O$ zumbido neurossensorial possui fisiopatologia ainda não muito conhecida. Sua etiopatogenia e fisiopatologia estão estreitamente relacionadas com as que causam surdez neurossensorial e tontura ${ }^{5}$.

A vertigem concomitante com o zumbido é um forte indício de alteração vestibular e a associação de tontura não rotatória com alterações neurovegetativas é indicativo de ausência de alteração vestibular ${ }^{6}$. Ambos os sintomas devem fazer parte da(s) 
mesma(s) etiologia(s), a(s) qual(is) deve(m) ser tratada(s) especificamente ${ }^{\text {? }}$.

O zumbido pode afetar a vida pessoal, profissional e familiar de alguns pacientes, comprometendo de forma importante o equilíbrio emocional ${ }^{8}$. Pessoas com zumbido de características psicoacústicas similares, podem apresentar reações opostas em relação ao zumbido ${ }^{9}$.

Como já descrito, o número de idosos com queixas de tontura e/ou zumbido é bastante freqüente. Um idoso que sente tontura por muitas vezes durante anamnese refere medo, angústia, às vezes receio em sair de casa sozinho, pois apresenta medo de cair na rua. Às vezes chega para a realização do exame com algum tipo de seqüelas, como hematomas e dores pelo corpo devido a tombos.

Existem diversos estudos na literatura nacional e internacional que descrevem o medo da queda em pacientes idosos ${ }^{10-13}$.

Os pacientes podem apresentar riscos significativos de queda se não tiverem a amplitude distal adequada de movimentos nos pés ${ }^{11}$.

A queda é um problema comum em idosos e se constitui uma das principais ameaças a sua independência. Acomete de 30 a $40 \%$ dos indivíduos acima de 65 anos na comunidade e cerca de 50\% dos indivíduos que vivem em casa de longa permanência ${ }^{10}$.

Os pacientes com tontura crônica apresentam prejuízos na qualidade de vida, em relação aos aspectos físicos, funcionais e emocionais ${ }^{14-16}$.

As alterações próprias do envelhecimento nos sistemas relacionados ao equilíbrio corporal, a maior possibilidade de doenças crônico-degenerativas e o uso crônico, por vezes múltiplo, de medicamentos, entre outros fatores, podem favorecer o aparecimento do sintoma tontura ou agravar a intensidade deste sintoma, provocando maior limitação física, funcional ou emocional nesta faixa etária ${ }^{14}$.

Durante a anamnese o examinador possui uma noção do que o paciente vem sofrendo, e por muitas das vezes o sentimento de angústia ${ }^{17,18}$. A anamnese é essencial; o paciente deve ser estimulado a descrever com suas próprias palavras o que sente e a influência dos sintomas nas suas atividades e na sua qualidade de vida ${ }^{19}$.

Outra etapa importante é a avaliação, esta deve ser criteriosa, pois abrange diversos fatores que possam estar determinando o desequilíbrio nestes pacientes. Vários problemas não vestibulares afetam o equilíbrio do paciente idoso, como doenças neurológicas, instabilidades cardiovasculares, doenças cerebrovasculares, distúrbios músculoesqueléticos, problemas visuais, doenças metabólicas, drogas terapêuticas e fatores psicológicos ${ }^{20}$.
A vertigem e a tontura são sintomas comuns que podem estar envolvidos no comprometimento de múltiplos sistemas e diferentes síndromes ${ }^{21}$. Sua etiologia é multifatorial e está relacionada com as mudanças estruturais e fisiológicas do aparelho vestibular em idosos ${ }^{22}$.

Devido à alta sensibilidade do sistema vestibular, é freqüente a relação de sintomas vestibulares com alterações específicas em outros órgãos ou sistemas, principalmente com o sistema metabólico, já que a orelha interna despende muita energia para o seu adequado funcionamento ${ }^{23}$. Incluem-se neste quadro de doenças metabólicas: Diabetes Mellitus, hiperinsulinemia ou hipoglicemia, hipertrigliceridemia, hiperlipidemias e alterações metabólicas decorrentes da insuficiência renal crônica ${ }^{24,25}$.

É importante investigar as alterações não labirínticas, pois, se o fator agressor do aparelho vestibular for mantido ou repetido, poderá provocar danos nesse sistema. Uma vez não sanada a doença de base, o paciente poderá apresentar crises labirínticas repetidas prejudicando a compensação central ${ }^{23}$.

Existem diversas enfermidades que podem causar a vertigem, como causas mais freqüentes, descreve-se:

- Neurite vestibular: Uma inflamação do labirinto, que se atribui a uma inflamação idiopática do nervo vestibular (porção do VIII par craniano que inerva o vestíbulo) ${ }^{26}$.

- Vertigem postural paroxística benigna: Caracteriza-se por acessos de vertigem de alguns segundos de duração predominantemente ao deitar-se, ao levantar-se ou em posição de decúbito ao girar a cabeça ${ }^{26}$.

- Síndrome de Meniére: Classicamente descrita como crises recorrentes de vertigem e hipoacusia unilateral, com duração de algumas horas, precedido de uma sensação de pressão e zumbido no ouvido afetado ${ }^{26}$.

- Vertigem vascular: Pode atribuir-se a alterações na vasorregulação cerebral. Ás vezes pode ser acompanhado de visão borrada, escurecimento da visão ${ }^{26,27}$.

- Insuficiência vertebro-basilar: Provocam uma diminuição transitória do fluxo sanguíneo das artérias vertebrais devido a perda de flexibilidade das mesmas ${ }^{26}$.

A importância deste estudo é justificada por que a cada ano vem aumentando a expectativa de vida dos indivíduos acima de 60 anos de idade. Estimase que o número de cidadãos idosos no Brasil aproxime-se de dez milhões de habitantes. As estatísticas referem que em 2005 o Brasil estaria entre os dez paises com o maior número de idosos ${ }^{28}$.

Atualmente os idosos procuram uma melhora na qualidade de vida, integrando-se, por exemplo, aos 
chamados grupos da terceira idade. Para tanto é necessário ter um equilíbrio corporal, psicológico e sentir-se bem dentro da sociedade. Um idoso saudável, inserido em sua comunidade pode desempenhar melhor as suas atividades.

O presente trabalho tem como objetivo realizar um estudo de prova calórica em pacientes idosos com queixas de tontura acompanhadas ou não de zumbido, com a finalidade de traçar um perfil dos idosos quanto os sintomas vestibulares.

\section{MÉTODOS}

A pesquisa foi realizada no setor de otoneurologia em uma clínica de Otorrinolaringologia na cidade de Niterói, no Rio de Janeiro. O estudo resulta de uma análise retrospectiva, a partir de pacientes idosos ${ }^{29} \mathrm{com}$ queixa de tontura.

Todos os indivíduos que participaram foram encaminhados por médicos para a realização do exame vestibular, em razão da queixa de tontura.

Foram selecionados 143 pacientes dos sexos feminino e masculino, com faixa etária variando de 60 a 90 anos de idade. Inicialmente analisou-se as questões retiradas da anamnese com o objetivo de selecionar somente idosos com queixas de tontura e tontura associada a zumbido. Foram excluídos desse estudo os idosos com queixa de desequilíbrio e aqueles idosos que apresentam queixas de zumbido desacompanhado da tontura.

Todos os pacientes foram submetidos ao exame otoneurológico, porém fará parte do corpus de dado desta pesquisa, somente os obtidos na prova calórica. Foram utilizados aparelho de vectoeletronistagmografia da marca Berger e o otocalorímetro da Contronic. A estimulação foi realizada com água a $44^{\circ} \mathrm{C}$ e $30^{\circ} \mathrm{C}$, e quando necessária a estimulação máxima de $18^{\circ} \mathrm{C}$.

A presente pesquisa submetida ao Comitê de Ética em Pesquisa do CEFAC - Saúde e Educação; sendo aprovada com $\circ \mathrm{n}^{\circ} 134 / 06$, julgada como sem risco e com necessidade do consentimento livre e esclarecido

Foram realizadas análises descritivas dos dados obtidos, focando o estudo da prova calórica em pacientes idosos com queixas de tonturas acompanhadas ou não de zumbido. Os métodos estatísticos utilizados foram a amostragem estratificada, observação das freqüências absolutas e relativas e representação gráfica dessas freqüências pelo gráfico de setores.

\section{RESULTADOS}

A idade média no grupo de idosos que fizeram parte da pesquisa foi de 69,76 anos, com desvio padrão de 7.0, valor mínimo de 60 anos e máximo de 90 anos.

Iniciou-se a análise dos resultados a partir da anamnese. Constatou-se que $71,1 \%$ dos sujeitos da pesquisa fazem uso de medicamentos, cardiovasculares e antidiabéticos. Outro dado encontrado com freqüência é a queixa de dificuldade de ouvir, relatada por $45 \%$ dos pacientes e a ocorrência de quedas, sofrida por $33 \%$ dos idosos (Tabela 1).

$\mathrm{Na}$ Tabela 2, são descritos os resultados absolutos em relação a VACL (velocidade angular da componente lenta). Sendo dividida em: normorreflexia (valores entre 3 e $51^{\circ} / \mathrm{seg}$ ), hiporeflexia (abaixo de 3 ㅇ /seg) e hiperreflexia (acima de 51이seg). Em relação aos resultados obtidos, nos valores absolutos, foram encontrados predomínios de normorreflexia.

Tabela 1 - Análise dos resultados obtidos na anamnese

\begin{tabular}{cccc} 
& Medicação & Quedas & Dificuldade Auditiva \\
\hline Sim & $n=102(71,1 \%)$ & $n=47(33 \%)$ & $n=66(45,8 \%)$ \\
Não & $n=41(28,9 \%)$ & $n=96(67 \%)$ & $n=77(54,2 \%)$ \\
\hline
\end{tabular}

$\mathrm{n}=$ número de sujeitos

Tabela 2 - Proporção dos resultados em cada tipo de teste

\begin{tabular}{|c|c|c|c|c|c|c|c|c|}
\hline & \multicolumn{4}{|c|}{$44^{\circ} \mathrm{C}$} & \multicolumn{4}{|c|}{$30^{\circ} \mathrm{C}$} \\
\hline & \multicolumn{2}{|c|}{ OD } & \multicolumn{2}{|c|}{ OE } & \multicolumn{2}{|c|}{ OD } & \multicolumn{2}{|c|}{ OE } \\
\hline & $\mathbf{N}$ & $\%$ & $\mathbf{N}$ & $\%$ & $\mathbf{N}$ & $\%$ & $\mathbf{N}$ & $\%$ \\
\hline Normorreflexia & 127 & $90,1 \%$ & 116 & $82,3 \%$ & 130 & $91,2 \%$ & 126 & $89,4 \%$ \\
\hline Hiporreflexia & 14 & $9,9 \%$ & 24 & $17 \%$ & 9 & $6,4 \%$ & 12 & $8,5 \%$ \\
\hline Hiperreflexia & 0 & $0 \%$ & 1 & $0,7 \%$ & 2 & $1,4 \%$ & 3 & $2,1 \%$ \\
\hline
\end{tabular}

OD: Orelha direita; OE: Orelha esquerda 
Tabela 3 - Análise dos resultados da prova calórica em valores absolutos de VACL

\begin{tabular}{|c|c|c|c|c|c|c|}
\hline & $44^{\circ} \mathrm{C}$ OD & $44^{\circ} \mathrm{C}$ OE & $30^{\circ} \mathrm{C}$ OD & $30 \circ \mathrm{C}$ OE & $18^{\circ} \mathrm{C}$ OD & $18^{\circ} \mathrm{C}$ OE \\
\hline Média & 9.411 & 7.44 & 16.9 & 18.74 & 12.06 & 9.118 \\
\hline Mediana & 8 & 7 & 16 & 17 & 11 & 9 \\
\hline Desvio Padrão & 7.061 & 6.924 & 11.029 & 12.89 & 11.149 & 7.688 \\
\hline Máximo & 40 & 55 & 54 & 50 & 36 & 24 \\
\hline Mínimo & 0 & 0 & 0 & 0 & 0 & 0 \\
\hline
\end{tabular}

OD: Orelha direita; OE: Orelha esquerda

A Tabela 3 demonstra a variabilidade dos resultados dos valores absolutos nas temperaturas utilizadas na prova calórica.

Constatou-se, a respeito dos resultados referentes a queixas (Figura 1), que a maioria dos idosos evidenciados na pesquisa, apresentam a queixa de tontura acompanhada de zumbido.

Com relação à faixa etária de indivíduos que sofreram quedas (Figura 2), pode-se observar, maior incidência entre aqueles de 70 a 79 anos.

Verificou-se que na análise dos resultados da prova calórica em valores relativos (Figura 3) um alto índice de resultados normais, com predomínio labiríntico ou predomínio da direção do nistagmo $<33 \%$.

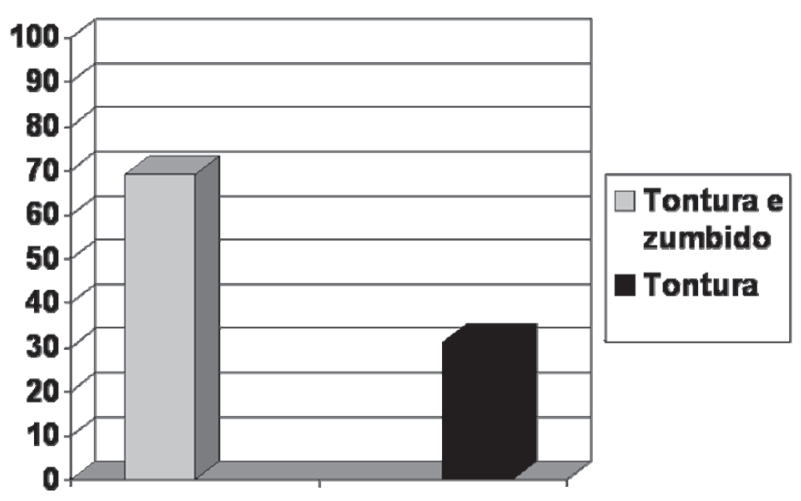

Figura 1 - Percentual de queixas apresentadas na pesquisa

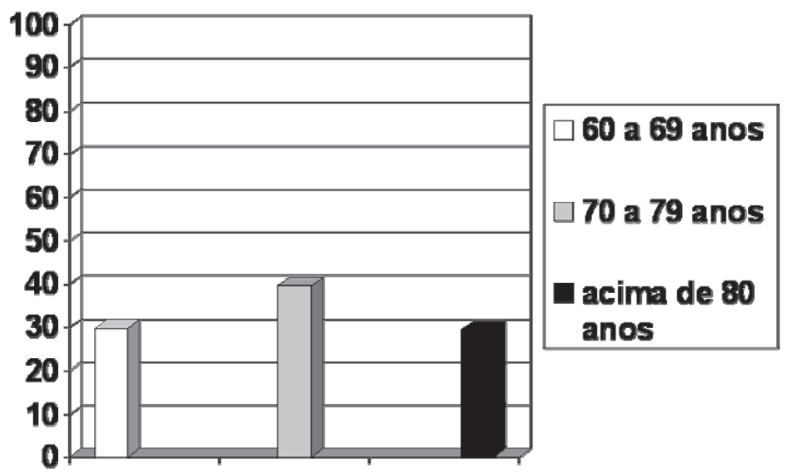

Figura 2 - Proporção de idosos que sofreram queda em cada faixa etária

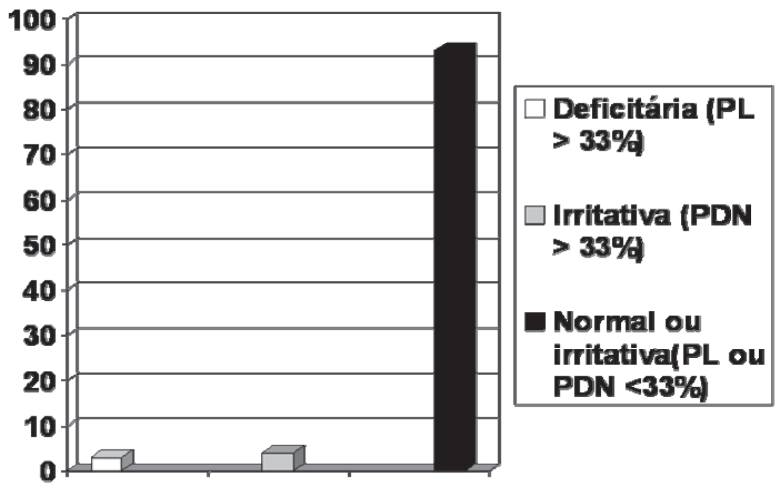

Figura 3 - Percentual referente aos resultados de prova calórica em valores relativos

No presente estudo levou-se em consideração a prova calórica, portanto, o número de pacientes considerados como resultado irritativo poderia ser maior, pois se houver alguma etapa do exame vestibular alterado, será considerado irritativa, mesmo com PL ou PDN < 33\%.

\section{DISCUSSÃO}

Sintomas como tonturas associadas ou não com o zumbido são muito comuns, principalmente na faixa etária abordada na pesquisa.

No presente estudo, $69 \%$ dos pacientes idosos com queixas de tonturas apresentam também o zumbido.Os resultados se assemelham aos encontrados em outra pesquisa ${ }^{30}$; no qual o grupo de sujeitos pesquisados foi dividido em dois grupos, sendo o grupo A formado por 40 idosos que realizavam atividades diversas semanalmente, sem alteração de equilíbrio e grupo $B$ com 40 idosos com queixas efetivas de alteração no equilíbrio corporal. Neste estudo em relação à presença de zumbido foi encontrado: $47,5 \%$ no grupo $A$ e $70 \%$ no grupo $B$.

Em relação à Tabela 1, fica evidente o número significativo de indivíduos $(71,1 \%)$ que fazem uso de medicação. $O$ fácil acesso à medicação e a baixa freqüência de uso de recursos não farmacológicos para o manejo de problemas médicos contri- 
bui para esse consumo elevado de medicamentos pela população de idosos. Como as doenças cardiovasculares vêm liderando as causas de morbimortalidade em indivíduos com idade acima de 65 anos, os medicamentos cardiovasculares têm sido amplamente prescritos pelos médicos ${ }^{31}$.

Ao relacionar a existência da polifarmácia nos idosos que vivem na comunidade com o número aumentado de medicamentos cardiovasculares e antidiabéticos, pode-se inferir que a exposição a múltiplos medicamentos nessa população, em sua maioria está vinculada a tratamentos de longo-prazo para doenças crônicas (doenças cardiovasculares, hipertensão, diabetes). Isto sugere relativa continuidade desse padrão de uso de medicamentos para os anos subseqüentes ${ }^{31}$.

Verificou-se que $33 \%$ dos pacientes participantes desta pesquisa, já haviam sofrido quedas. Este dado vai ao encontro da literatura ${ }^{10}$. As quedas são as conseqüências mais perigosas do desequilíbrio e da dificuldade de locomoção, sendo seguidas por fraturas, deixando os idosos acamados por dias e sendo responsáveis por $70 \%$ das mortes acidentais em pessoas com mais de 75 anos ${ }^{30}$.

Em relação à distribuição dos idosos segundo a presença ou não de dificuldade de audição, 45,8\% referiram na anamnese este dado. Talvez este número seria maior se fosse verificado no estudo, a audiometria realizada. Na pesquisa foi relatada apenas a queixa deste paciente sem verificar a configuração audiométrica.
Com relação à queixa (Figura 1), houve um grande percentual de idosos que relataram a tontura acompanhada de zumbido, fato este justificado pelo processo fisiológico do envelhecimento.

Verifica-se pela Tabela 2, que a maior parte dos pacientes submetidos à prova calórica, apresentam resultados em valores absolutos em normorreflexia (3 a 51/seg) e na Figura 3, um percentual grande dos pacientes idosos com valores relativos de $\mathrm{PL}$ ou PDN $<33 \%$, sendo considerados normais. A literatura informa que as mudanças na função vestibular foram comparadas com um déficit vestibular bilateral progressivo ${ }^{11}$, porém nesta pesquisa fica evidente a prevalência dos resultados normais em valores absolutos e relativos. Em outros estudos foram encontrados resultados similares 0 da presente pesquisa ${ }^{32,33}$.

Houve presença de EIFO em todos os exames e não foi observado sinal patognomônico de alteração central.

\section{CONCLUSÃO}

Mediante análise dos dados, o presente estudo nos permitiu concluir que houve diferença estatisticamente significativa entre pacientes com a queixa tontura associada ao zumbido, comparado aqueles apenas com queixa de tontura e, em relação à prova calórica, o trabalho demonstrou a alta prevalência de pacientes com valores absolutos e relativos normais.

\begin{abstract}
Purpose: to accomplish the study of caloric testing in senior patients with dizziness, accompanied or not of with buzzing. Methods: 143 patients were analyzed with age varying from 60 to 90 years; all of them did an otoneurologic evaluation. Senior patients with unbalance complaints and with buzzing unaccompanied with dizziness were excluded from this study. Results: $82.3 \%$ to $91.2 \%$ of the patients obtained normoreflexia in absolute values and over $93 \%$ obtained normal results in relative values. Referring to the data obtained in the anamnesis, what got attention was $69 \%$ of dizziness accompanied with buzzing. And the high medication of the patients in this age group. Conclusion: there was a prevalence of dizziness accompanied with buzzing in the studied population. Concerning the caloric testing, the study demonstrated the high prevalence of patients with normal absolute and relative values.
\end{abstract}

KEYWORDS: Dizziness; Vertigo; Aged; Vestibular Functions Tests; Tinnitus 


\section{REFERÊNCIAS}

1. Ganança MM, Caovilla HH. Desequilíbrio e reequilíbrio. In: Ganança MM, organizador. Vertigem tem cura? São Paulo: Lemos; 1998. p.13-9.

2. Honrubia V. Testes quantitativos da função vestibular e o exame clínico. In: Herdman SJ. Reabilitação vestibular. São Paulo: Manole; 2002. p. $105-62$.

3. Campos CAH. Principais quadros clínicos no adulto e no idoso. In: Ganança MM, editor. Vertigem tem cura? São Paulo: Lemos; 1998. p.49-57.

4. Sanchez TG, Netto BM, Sasaki F, Santoro PP, Bento RF. Zumbido gerado por alterações vasculares e musculares. Arq Fund Otorrinolaringol. 2000; 4(4):136-42.

5. Fukuda Y. Zumbido e suas correlações otoneurológicas. In: Ganança MM. Vertigem tem cura? 1. ed. São Paulo: Lemos; 1998.p.171-6.

6. Jardim M, Ferreira VJA. Identificadores sintomatológicos da síndrome vestibular periférica. Rev CEFAC. 2002; 4(3):207-12.

7. Menon AD, Sakano E, Weckx LLM. Vertigem e zumbido: consenso sobre vertigem. Rev Bras Otorrinolaringol. 2000; 66(6):S11.

8. Coelho CCB, Sanchez TG, Bento RF. Características do zumbido em pacientes atendidos em serviço de referência. Rev Arq Otorrinolaringol. 2004; 8(3):216-24.

9. Gomes SJV, Barboza RM, Santos TMM. A incidência de zumbido numa mostra aleatória na cidade de Salvador. Rev CEFAC. 2004; 6(1):89-93. 10. Maciel AC, Scoralick FM. Epilepsia atônica como causa de quedas em uma paciente idosa. J Bras Med. 2005; 88(3):1-3.

11. Whitney SL. Tratamento do idoso com disfunção vestibular. In: Herdman SJ. Reabilitação vestibular. São Paulo: Manole; 2002.p. 505-28.

12. Shobha SR. Prevention of falls in older patients. [periódico online]. Physician. 2005. Disponível em: URL: http://www.findarticles. com/p/articles/mi-m3225/is-1-72/ai-n14787793/

13. Neil Cherian MD. Dizziness. 2006. [periódico online]. Disponível em: URL: http://www. clevelandclinicmed.com/diseasemanagement/ neurology/dizziness.htm.

14. Ganança FF, Castro ASO, Branco FC, Natour J. Interferência da tontura na qualidade de vida de pacientes com síndrome vestibular periférica. Rev Bras Otorrinolaringol. 2004; 70(1):94-101.

15. Matheson A, Darlington C, Smith PF. Dizziness in the elderly and age-related degeneration on the vestibular system. [periódico online] New Zealand J Psychol. 1999. Disponível em: URL: http://www.findarticles. com/p/articles/mi-qa3848/is-199906/ai-n8856160
16. Colledge NR, Wilson JA, Mancintyre CCA, Maclenann WJ. The prevalence and characteristics of dizziness in an elderly community. [periódico online] Age and Ageing. 2004. Disponível em URL: http://ageing.oxfordjournals. org/cgi/content/abstract/23/2/117.

17. Kuhn AMB, Casagrande MC. A contribuição da avaliação psicológica. In: Ganança MM. Vertigem tem cura? São Paulo: Lemos; 1998. p.127-34.

18. Paiva AD, Kuhn AMB. Sintomas psicológicos concomitantes à queixa de vertigem em 846 prontuários de pacientes de otoneurológicos do Ambulatório de Otoneurologia da Universidade Federal da São Paulo - Escola Paulista de Medicina. Rev Bras Otorrinolaringol. 2004; 70(4):512-5.

19. Ganança MM, Caovilla HH. Universo das tonturas. In: Ganança MM, organizador. Vertigem tem cura? São Paulo: Lemos; 1998. p. 21-6.

20. Menon AD, Sakano E, Weckx LLM. Tratamento do paciente idoso. Consenso sobre vertigem. Rev Bras Otorrinolaringol. 2000; 66(6):31.

21. Kanashiro AMK, Pereira CB, Melo ACP, Scaff M. Diagnóstico e tratamento das principais síndromes vestibulares. Arq Neuropsiquiatr. 2005; 63(1):140-4.

22. Chavez RG, Cordenas VI. Detection de câmbios por envejecimiento de labirinto posterior mediante eletro-oculografia. Ann Otorrinolaringol. 2004. 46(2):60-2.

23. Fernandes JR, Ganança CF. Avaliação vestibular analógica e computadorizada em pacientes com distúrbios de origem metabólica. Arq Otorrinolaringol. 2004; 8(3):208-15.

24. Tiensoli LO, Couto ER, Mitre EI. Fatores associados à vertigem ou tontura em indivíduos com exame vestibular normal. Rev CEFAC. 2004. 6(1):94-100.

25. Scherer LP, Lobo MB. Pesquisa do nistagmo/vertigem de posição e avaliação eletronistagmográfica em um grupo de indivíduos portadores de diabetes mellitus tipo I. Rev Bras Otorrinolaringol. 2002; 68(3):355-60.

26. Murillo FG, Pineda ZMV. Vertigo: uma visión otorrinolaringológica para la medicina general. Acta Méd. 2002. 44; 1. [periódico online] Disponível em: URL: http://www.scielo.sa.cr/scielo.php?script=sci arttext\&pid=S0001- 60022002000100003\&lng=pt\& nrm=iso>. ISSN 0001-6002.

27. Ruiz IF. Vertigo de origén vascular. Rev Med Electron. 2004. 26; 2. [periódico online] Disponível em URL: http://cpimtz.sed.cu/revista\%20medica/ ano/202004/vol2\%202004/tema08.htm.

28. Mota PH, Franco ES, Pinto ESM, Arieta AM. Estudo do equilíbrio no idoso por meio de eletronistagmografia. Acta Awho. 2002; 21:3. 
29. Brasil. Estatuto do Idoso. Lei n.10741 de 1 de Outubro de 2003. Artigo 1‥

30. Ruwer SL, Rossi AG, Simon LF. Equilíbrio no idoso. Rev Bras Otorrinolaringol. 2005; 71(3):298-303.

31. Flores LM, Mengue SS. Uso de medicamentos por idosos em região do sul do Brasil. Rev Saúde Publ. 2005; 39(6):924-9.
32. Gushikem P, Caovilla HH, Ganança MM. Avaliação otoneurológica em idosos com tontura. Acta Awho. 2002; 21(1):25.

33. Fukuda C, Silva AM, Gushikem P, Caovilla $\mathrm{HH}$, Ganança MM. Avaliação otoneurológica em indivíduos idosos: achados clínicos audiológicos e vestibulares. In: 35o Congresso Brasileiro de Otorrinolaringologia, Natal; 2000. p.133.

RECEBIDO EM: 09/04/2007

ACEITO EM: 22/04/2008

Endereço para correspondência:

Av. Brasil, 1020 c 04

Duque de Caxias - RJ

CEP: 25065-170

Tel: (21) 3712-4452 / (21) 9951-9758

E-mail: dcobrj@ig.com.br 